\title{
STEEPER, FLATTER, OR JUST "SALPETER"? EVIDENCE FROM GALAXY EVOLUTION AND GALAXY CLUSTERS
}

\author{
Alvio Renzini \\ ESO, Garching, Germany \\ arenzini@eso.org
}

\begin{abstract}
A single-slope Salpeter IMF overpredicts the stellar $M_{*} / L_{\mathrm{B}}$ ratio by about a factor of 2 , which requires the IMF to be flatter below $\sim 1 M_{\odot}$. On the other hand a Salpeter IMF for $M \gtrsim M_{\odot}$ predicts an evolution with redshift of the fundamental plane of ellipticals in clusters which is in agreement with the observations and a formation at $z \gtrsim 3$ for these galaxies. A Salpeter IMF for $1 \lesssim M \lesssim 40 M_{\odot}$ also predicts the observed amount of heavy elements (oxygen and silicon) in clusters of galaxies.
\end{abstract}

\section{Introduction}

The most direct way of measuring the IMF is by stellar counts, a technique that can be applied only within a limited distance from us. On the other hand, much of galaxy properties - at low as well as at high redshift - depend on the IMF, including mass-to-light ratios, derived star-formation rates, feedback, metal enrichment, etc. Considering these properties enables us to gather strong constraints on the IMF for stellar systems and astrophysical situations that cannot be probed by stellar counts, and provides sanity checks for assumptions and theories of the IMF. In this talk I present the constraints on the IMF slope below $\sim 0.5 M_{\odot}$, between $\sim 1$ and $\sim 1.4 M_{\odot}$, and between $\sim 1$ and $\sim 25 M_{\odot}$ that come respectively from the observed values of the $M_{*} / L_{\mathrm{B}}$ ratio of local elliptical galaxies, from the redshift evolution of the fundamental plane, and from the observed mass of heavy elements in clusters of galaxies. I will also emphasize the role played by the IMF in our attempts at mapping the cosmic history of star formation, and the build up of the overall stellar mass content of the universe. The parameters of the con- 
cordance cosmology are adopted throughout this paper, i.e., $\Omega_{\mathrm{m}}=0.3$; $\Omega_{\Lambda}=0.7$, and $H_{\circ}=70$.

\section{The Mass-to-Light Ratio of Ellipticals and the IMF Slope Below $\sim 1 M_{\odot}$}

Fig. 1 shows as a function of age the $M_{*} / L_{\mathrm{B}}$ ratio for simple stellar populations (SSP), i.e., assembly of coeaval stars all of the same (solar) chemical composition. SSP models are from Maraston (1998), while the use of other models would give essentially the same result. The mass-to-light ratio varies wildly depending on the assumed IMF, which in this case is described by a single slope power law $\phi(M) \propto M^{-s}$ for $0.1<M<100 M_{\odot}$, where $s=2.35$ corresponds to the Salpeter IMF (Salpeter 1955). Note that very large mass-to-light ratios are obtained for either very steep $(s=3.35)$ or very flat $(s=1.35)$ IMFs. In the former case the total stellar mass is dominated by the huge number of low mass stars, i.e., the IMF is dwarf dominated. In the latter case instead (at late times) most stellar mass is locked into remnants: black holes, neutron stars and white dwarfs, while low mass stars contribute very little to the mass budget, i.e., the top-heavy IMF leads to a remnant dominated mass. Of the three choices, the Salpeter slope gives the lowest values of $M_{*} / L_{\mathrm{B}}$. Yet, at an age of $\sim 12 \mathrm{Gyr}$, appropriate for the the bulk stellar population of elliptical galaxies ${ }^{1}$, the $M_{*} / L_{\mathrm{B}}$ ratio is a factor $\sim 2$ too high compared to the stellar mass of ellipticals as derived dynamically (e.g. van der Marel 1991). This excludes any single-slope IMF, hence the IMF must flatten with respect to the Salpeter value below $\sim 0.5-0.7 M_{\odot}$, as indeed indicated by all direct stellar count in Disk and Bulge fields and in stellar clusters alike, and extensively reported at this meeting. For example, assuming $s=2.35$ for $M>0.6 M_{\odot}$ and $s=1.35$ for $M<0.6 M_{\odot}$ the $M_{*} / L_{\mathrm{B}}$ ratio is reduced by about a factor of 2 , thus bringing SSP models in agreement with the observed values.

\section{The Redshift Evolution of the Fundamental Plane, and the IMF Slope for $1 \lesssim M \lesssim 1.4 M_{\odot}$}

As is well known, elliptical galaxies tightly cluster around a plane in the space having for coordinates the central velocity dispersion $(\sigma)$, the effective radius $\left(R_{\mathrm{e}}\right)$ and the effective surface brightness $\left(\mu_{\mathrm{e}}\right)$ (Djorgovski \& Davis 1987; Dressler et al. 1987). This fundamental plane (FP)

\footnotetext{
${ }^{1}$ Compelling evidence has accumulated over the years indicating that the star-formation process in elliptical galaxies was virtually complete by $z \simeq 3$, corresponding to an age of $\sim 12$ Gyr (for an extensive review see Renzini 1999).
} 


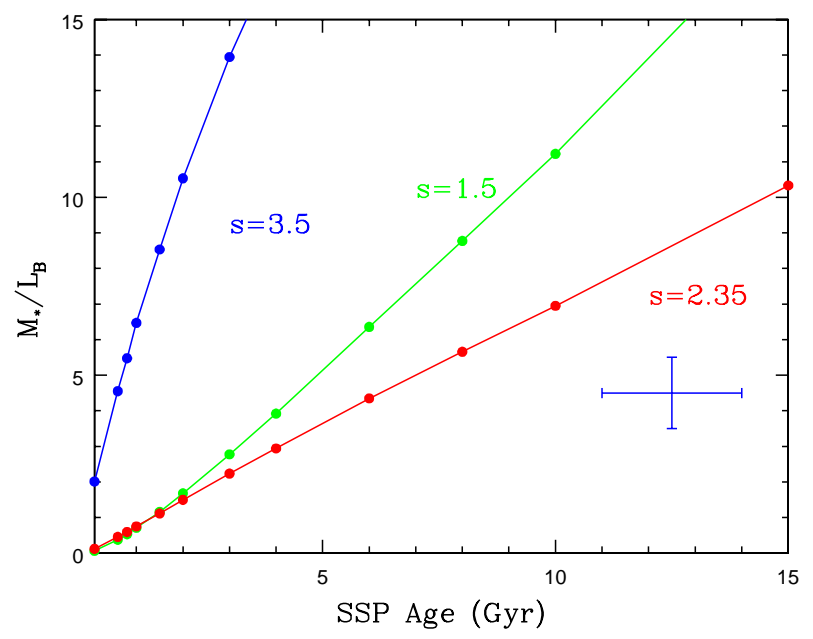

Figure 1. The time evolution of the stellar $M_{*} / L_{\mathrm{B}}$ ratio for solar metallicity simple stellar populations with various slopes $(s)$ of the IMF (synthetic models from Maraston 1998). The cross on the right side of the figure gives the typical ranges for the $M_{*} / L_{\mathrm{B}}$ ratio of local elliptical galaxies and for the ages of their dominant stellar populations.

combines two structural/dynamical quantities $\left(\sigma\right.$ and $\left.R_{\mathrm{e}}\right)$ with the third $\left(\mu_{\mathrm{e}}\right)$ which instead depends on luminosity, hence on the stellar population content of the galaxy and in particular on its age.

The slope of the IMF controls the rate of luminosity evolution of a SSP. The flatter the IMF the faster the luminosity decline past a burst of star formation. On the contrary, the steeper the IMF the slower such decline, as the light from the larger number of low-mass stars compensates for the progressive death of the more massive stars. As we look to higher and higher redshift ellipticals, we therefore expect them to depart from the local FP by an amount that depends on the slope of the IMF. Thus, if small ellipticals were to have a different IMF slope w.r.t. large ellipticals, with increasing redshift one would expect the small ellipticals to depart from the local FP by a different amount w.r.t. large ellipticals: the FP would rotate with increasing redshift (Renzini \& Ciotti 1993). Fig. 2 shows that ellipticals in a cluster at $z=0.58$ and another at $z=0.83$ (corresponding to a lookback time of $\sim 7 \mathrm{Gyr}$, or $\sim 1 / 2$ the age of the universe) align parallel to the FP defined by ellipticals in the COMA cluster (Wuyts et al. 2003): the FP does not rotate, hence there is no appreciable trend of the IMF with galaxy size, mass, or luminosity.

The shift in the FP shown in Fig. 2 actually corresponds to a change in the $M / L_{\mathrm{B}}$ ratio of the observed galaxies, and Fig. 3 shows such a change for the two mentioned clusters, plus a few others at various 


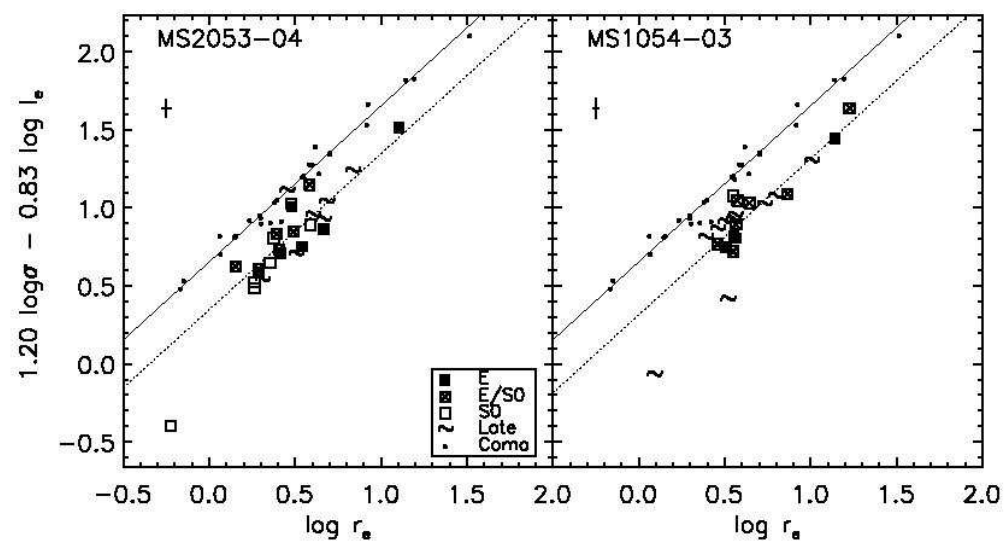

Figure 2. The fundamental plane of clusters MS2053-04 $(z=0.58)$ and MS1054-03 $(z=0.83)$ compared to the FP of Coma (Wuyts et al. 2003). Note that the slope of the FP appears to remain the same with increasing redshift, indicating that the slope of the IMF is independent of elliptical galaxy size, mass, or luminosity.

redshifts, including one at $z=1.27$ (van Dokkum \& Stanford 2003). ${ }^{2}$ Overplotted is also shown the evolution with redshift of the $M_{*} / L_{\mathrm{B}}$ ratio of SSP models (from Maraston 1998) having assumed the SSPs formed at $z_{\mathrm{F}}=5$. The various $M_{*} / L_{\mathrm{B}}$ ratios have been normalized to their value at $z=0$, so as vto emphasize their relative change with redshift.

At $z=0$ elliptical galaxies $\sim 12$ Gyr old harbour stellar populations with $\sim 1 M_{\odot}$ stars at the main sequence turnoff (MSTO). By $z=1.5$ the precursors of such populations have an age of only $\sim 3$ Gyr, and correspondingly a higher mass at the MSTO, but not by much so. Specifically, the MSTO mass at an age of $\sim 3 \mathrm{Gyr}$ is $\sim 1.4-1.5 M_{\odot}$, and therefore by following the evolution of the FP with redshift up to $z \sim 1.5$ (or equivalently of the mass-to-light ratio) we explore the IMF slope within the rather narrow mass interval $1 \lesssim M \lesssim 1.4 M_{\odot}$. In practice, we measure the slope of the IMF near $M=M_{\odot}$.

Note that in Fig. 3 a Salpeter IMF provides a decent, yet not perfect fit to the data. On the other hand, at each redshift the $M_{*} / L_{\mathrm{B}}$ ratio depends on the assumed age of the SSP, hence on the assumed redshift of formation $z_{\mathrm{F}}$. This means that to some extent the IMF slope and the formation redshift are degenerate. Indeed, the Salpeter IMF provides a better fit to the FP shifts if one assumes a lower formation redshift, e.g. $z_{\mathrm{F}}=3$, as illustrated in Fig. 4 .

${ }^{2}$ In the $z=1.27$ cluster $\sigma$ was measured for only two galaxies, and given the errors the slope of the FP could not be reliably measured, so only the zero-point shift is shown here. 


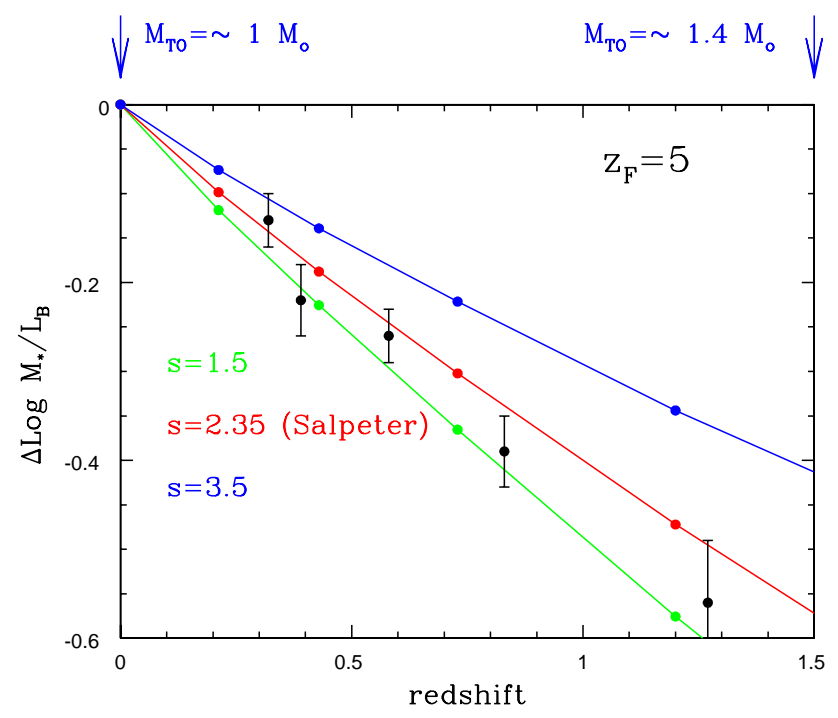

Figure 3. The evolution with redshift of the $M_{*} / L_{\mathrm{B}}$ ratio of simple stellar populations (SSP) of solar metallicity and various IMF slopes, normalized to its value at $z=0$. A formation redshift $z_{\mathrm{F}}=5$ is assumed for the SSPs. The lines refer to $s=1.5$ (top), 2.35 (middle) and 3.5 (bottom). The data points refer to the shifts in the fundamental plane locations for clusters of galaxies at various redshifts (from van Dokkum \& Stanford 2003). Note that for this assumed redshift of formation the stellar mass at the main sequence turnoff is $\sim 1.4 M_{\odot}$ at $z=1.5$ and $\sim M_{\odot}$ at $z=0$, as indicated by the arrows.

Of course, the higher the redshift, the larger the difference between the $M_{*} / L_{\mathrm{B}}$ ratios predicted by SSPs with different IMF slopes. Therefore, measuring the FP shift at the highest possible redshift would be of great interest. For quite many years the highest redshift elliptical galaxy known was LBDS 53w091 at $z=1.55$ (Dunlop et al. 1996; Spinrad et al. 1997), not much beyond the highest redshift ellipticals in the study by van Dokkum \& Stanford (2003), which are at $z=1.27$. However, passively evolving elliptical galaxies have now been identified all the way to $z=1.9$ (Cimatti et al. 2004), for which it would be very interesting to measure the central velocity dispersion, thus getting the FP parameters, although this may require extremely long integrations.

To further illustrate the case of the IMF slope/ $z_{\mathrm{F}}$ degeneracy, Fig. 5 shows the FP $\left(M_{*} / L_{\mathrm{B}}\right)$ shift with redshift for SSPs with a Salpeter IMF, solar metallicity, and various formation redshifts $z_{\mathrm{F}}$. One can conclude that the degeneracy is strong if the formation redshift of ellipticals were to be relatively low (e.g., $\sim 2$ ), but for $z_{\mathrm{F}} \gtrsim 3$ the degeneracy is quite mild and the data favor an IMF slope for $1 \lesssim M \lesssim 1.4 M_{\odot}$ very close to the Salpeter value. 


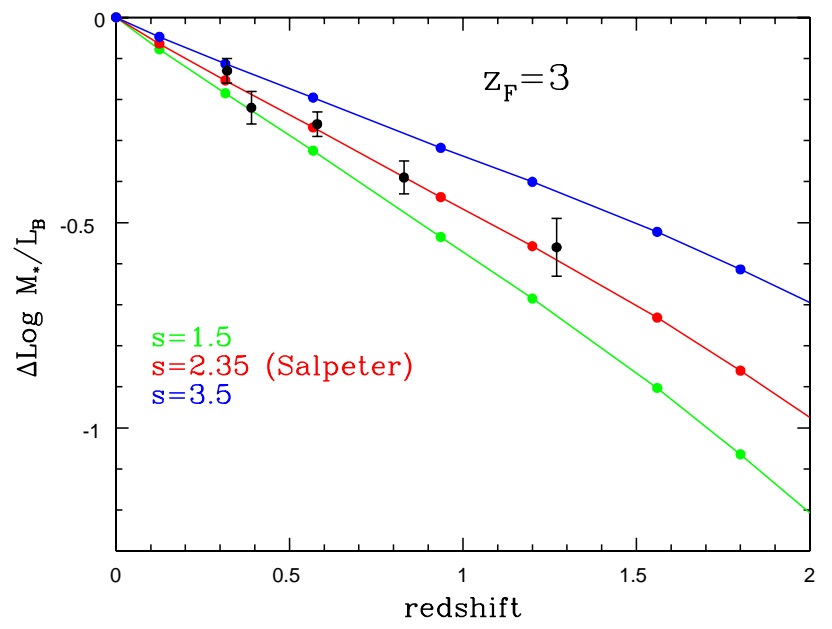

Figure 4. The same as in Fig. 3 but assuming a formation redshift $z_{\mathrm{F}}=3$.

\section{The Metal Content of Galaxy Clusters, and the IMF Slope for $1 \lesssim M \lesssim 25 M_{\odot}$}

In clusters of galaxies most of the light comes from early-type galaxies dominated by old stellar populations, i.e., from $\sim 1 M_{\odot}$ stars. Meanwhile, one can also measure the amount (mass) of metals contained in the intracluster medium and in the stars. As most metals are produced by Type II supernovae, the total metal mass in a cluster of galaxies is proportional to the number of massive stars $\left(M \gtrsim 8 M_{\odot}\right)$ that have exploded in the far past, therefore dispersing the metals we see today ${ }^{3}$. It follows that the metal-mass-to-light ratio of galaxy clusters (e.g., Renzini 2003) provides a measure of the number ratio of massive to $\sim M_{\odot}$ stars, i.e., of the IMF slope between $\sim 1$ and $\sim 25 M_{\odot}(\text { or more })^{4}$ for the stellar populations that - when young - have produced the metals and now - that are old - produce the light we see today.

The IMF of a passively evolving SSP can be written as:

$$
\phi(M)=a(t, Z) L_{\mathrm{B}} M^{-s}
$$

\footnotetext{
${ }^{3}$ Iron is the element whose abundance is most reliably measured both in the ICM and in galaxies. However, its production may be dominated by Type Ia supernovae, whose progenitors are binary stars. Given the complexities introduced by the binary nature of the iron producers, this element is less useful for the determination of the IMF slope.

${ }^{4}$ With a Salpeter IMF the typical stellar mass for nucleosynthesis yields is $\sim 25 M_{\odot}$, i.e., the overall yield can be approximated by the total number of Type II supernovae times the nucleosynthesis products of a $25 M_{\odot}$ star.
} 


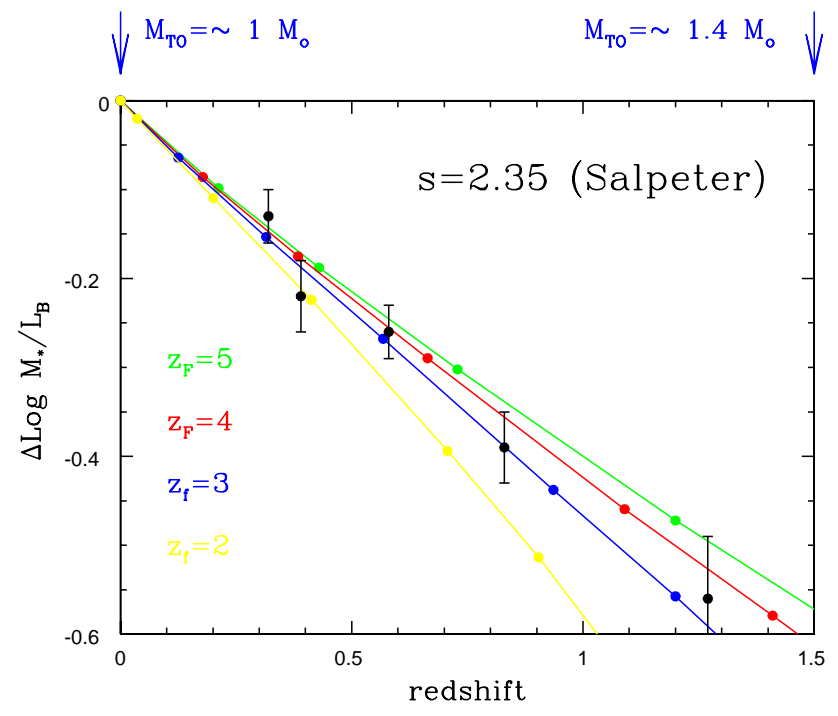

Figure 5. The same as in Fig. 3 for the Salpeter IMF and various values of the formation redshift $z_{\mathrm{F}}$, from $z_{\mathrm{F}}=5$ (top) to $z_{\mathrm{F}}=2$ (bottom).

where $L_{\mathrm{B}}$ is its $B$-band luminosity and $a(t, Z)$ is a (slow) function of the SSP age and metallicity. For example, for $Z=Z_{\odot}=0.02$ one has $a(t)=1.67$ and 2.51, respectively for $t=10$ and 15 Gyr (Maraston 1998) (with $M$ and $L_{\mathrm{B}}$ in solar units). More recent models (Maraston 2004, in preparation) give $a(t)=1.68$ and 2.8 for the same $(t, Z)$ and $a(t)=2.50$ and 3.94 for $Z=2 Z_{\odot}$. For $t=12$ Gyr (the minimum age for $z_{\mathrm{F}} \gtrsim 3$ ) the new Maraston models give $a(Z)=2.22$ and 3.12 , respectively for $Z=Z_{\odot}$ and $Z=2 Z_{\odot}$.

Therefore, the metal-mass-to-light ratio for the "X" element can be calculated in a straightforward manner from:

$$
\frac{M_{\mathrm{X}}}{L_{\mathrm{B}}}=a(t, Z) \int_{8}^{40} m_{\mathrm{X}}(M) M^{-s} d M
$$

where $m_{\mathrm{X}}(M)$ is the mass of the element "X" which is produced by a star of mass $M$. Adopting $a(t, Z)=3$ and $m_{\mathrm{X}}(M)$ for oxygen and silicon from Woosley and Weaver (1995) and integrating Eq. (2) one obtains the oxygen- and the silicon-mass-to-light ratios, which are shown in Fig. 6 as a function of the IMF slope. As expected, the $M_{\mathrm{O}} / L_{\mathrm{B}}$ and $M_{\mathrm{Si}} / L_{\mathrm{B}}$ ratios are extremely sensitve to the IMF slope. The values observed in clusters of galaxies (ICM plus galaxies) are $\sim 0.1$ and 


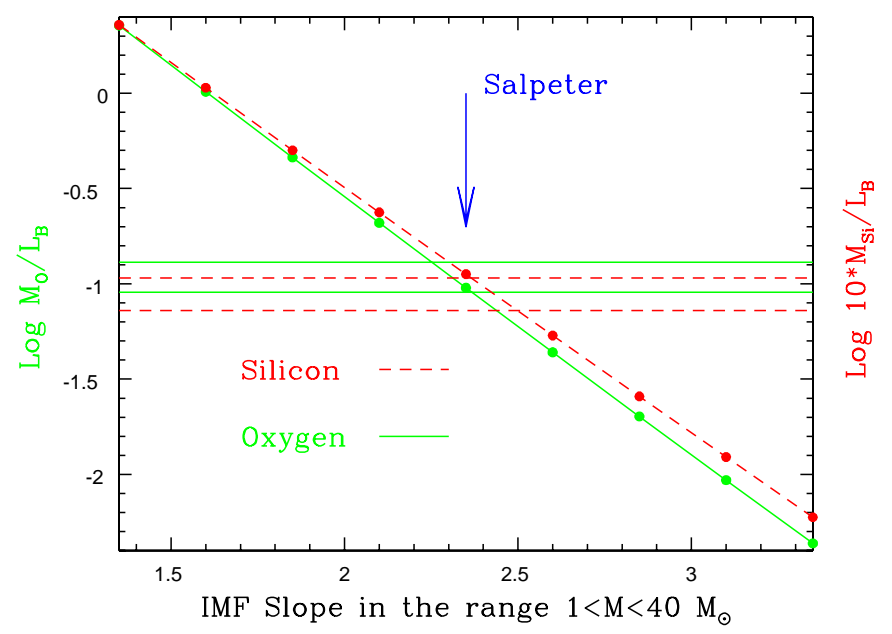

Figure 6. The Oxygen- and the Silicon-Mass-to-Light Ratios as a function of the IMF slope as calculated from Eq. (2) with $a(t, Z)=3$ and using the nucleosynthesis prescription of Woosley \& Weaver (1995). The horizontal lines show the observed average values of this ratios in clusters of galaxies, and their uncertainty range.

$\sim 0.01 M_{\odot} / L_{\odot}$, respectively for oxygen and silicon ${ }^{5}$ (e.g., Finoguenov et al. 2003; Portinari et al. 2004, and references therein). These empirical values are reported in Fig. 6, which at the meeting was presented to Ed Salpeter as a Happy-Birthday postcard. Indeed, with the Salpeter IMF slope $(s=2.35)$ the standard explosive nucleosynthesis from Type II supernovae produces just the right amount of oxygen and silicon to account for the observed $M_{\mathrm{O}} / L_{\mathrm{B}}$ and $M_{\mathrm{Si}} / L_{\mathrm{B}}$ ratios in cluster of galaxies, having assumed that most of the cluster $B$-band light comes from $\gtrsim 12$ Gyr stellar populations.

Fig. 6 also shows that with $s=1.35$ such a top heavy IMF (in various circumstances invoked to ease discrepancies between theories and observations) would overproduce metals by more than a factor of 20 . This is indeed the change one expects in $M_{\mathrm{O}} / L_{\mathrm{B}}, M_{\mathrm{Si}} / L_{\mathrm{B}}$, etc. for a $\Delta s=1$ when considering that the light $L_{\mathrm{B}}$ is provided by $\sim M_{\odot}$ stars and the metals by $\sim 25 M_{\odot}$ stars.

${ }^{5}$ These values result from averaging over the reported values for individual clusters with different ICM temperature, and take into account that $\sim 10-30 \%$ of the stellar mass in clusters is not bound to individual galaxies (Arnaboldi et al. 2003; Gal-Yam et al. 2003). 


\section{Masses and Star Formation Rates of High Redshift Galaxies}

As is well known, all SFR indicators (UV continuum, $\mathrm{H} \alpha$, sub-mm, etc.) measure the formation rate of massive stars. To derive the total SFR an IMF (slope and shape) has to be assumed. Similarly, an IMF must be assumed to derive the total stellar mass of a galaxy from its spectral energy distribution (SED) and luminosity. These are indeed determined by the number of stars producing the bulk of the light (which are typically in a quite narrow range of masses), while remnants and dwarfs produce little light but may contribute a major fraction of the mass. It follows that the time integral of the measured $\operatorname{cosmic} \operatorname{SFR}(z)$

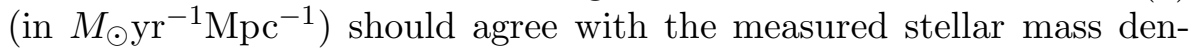
sity $\rho_{*}(z)$ (in $M_{\odot} \mathrm{Mpc}^{-1}$ ), and in turn at $z=0$ this should agree with its dynamical estimate. Clearly, such a general agreement needs to be achieved with the right value of the IMF. Fig. 7 shows a recent attempt in this direction (adopting a Salpeter IMF, Fontana et al. 2004) but error bars are still too large to allow a definitive answer. However, several galaxy surveys (with HST, SST, VLT, etc. etc.) are now well under way, and extremely rapid progress is expected in this field.

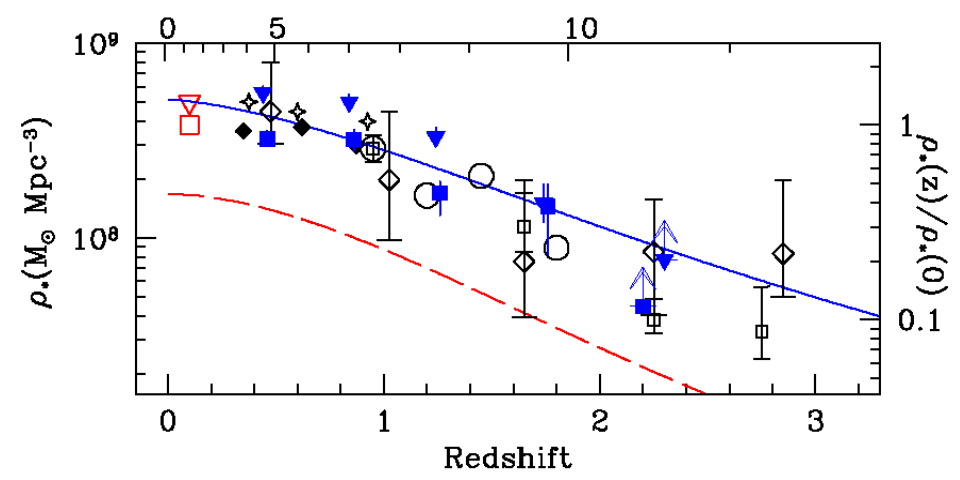

Figure 7. The cosmic evolution of the comoving density of the overall mass in stars from various current surveys, compared with the time integral of the cosmic starformation rate density, uncorrected (dashed line) and corrected for dust attenuation effects (solid line) (from Fontana et al. 2004).

\section{Summary}

$\star$ The IMF of elliptical galaxies is close to Salpeter except below $\sim 0.6 M_{\odot}$, where it flattens.

$\star$ The fundamental plane (FP) of ellipticals does not rotate with redshift, 
implying that the IMF is independent of galaxy luminosity (mass). $\star$ The FP shift with redshift is consistent with a Salpeter IMF near $M \sim M_{\odot}$ and a formation at $z \gtrsim 3$ for the bulk of stars in ellipticals.

$\star$ The overall metal content of galaxy clusters (ICM+galaxies) is well reproduced by a Salpeter IMF and classical nucleosynthesis.

$\star$ Very rapid progress is expected in determining the cosmic history of star formation along with the cosmic build-up of stellar mass and a consistent IMF.

$\star$ The IMF may not be universal (who knows??) but the one of which this meeting celebrates the 50th anniversary doesn't quite show its age (!).

Acknowledgments. I would like to thank Claudia Maraston for having provided her most recent models in advance of publication, and the staff of the Carnegie Observatories (Pasadena) for their kind hospitality during the time when this paper was written and set up.

\section{References}

Arnaboldi, M. et al. 2003, AJ, 125, 514

Cimatti, A., et al. 2004, Nature, 430, 184

Djorgovski, S., \& Davis, M. 1987, ApJ, 313, 59

Dressler, A. et al. 1987, ApJ, 313, 42

Dunlop, J.S., et al. 1996, Nature, 381, 581

Finoguenov, A., Burkert, A., \& Böhringer, H. 2003, ApJ, 594, 136

Fontana, A., et al. 2004, A\&A, 424, 23

Gal-Yam, A., et al. 2003, AJ, 125, 1087

Maraston, C. 1998, MNRAS, 300, 872

Portinari, L., et al. 2004, ApJ, 604, 579

Renzini, A., \& Ciotti, L. 1993, ApJ, 416, L49

Renzini, A. 1998, AJ, 115, 2459

Renzini, A. 1999, in The Formation of Galactic Bulges, ed. M. Carollo, H. Ferguson, \& R. Wise (Cambridge, CUP), p. 9 (astro-ph/9902108)

Renzini, A. 2004, in Clusters of galaxies: Probes of Cosmological Structure and Galaxy Evolution, ed. J.S. Mulchaey, A. Dressler, \& A. Oemler (Cambridge, CUP) (astro$\mathrm{ph} / 0307146)$

Salpeter, E. E. 1955, ApJ, 123, 666

Spinrad, H., et al. 1997, ApJ, 484, 581

van der Marel, R. 1991, MNRAS, 253, 710

van Dokkum, P.G., \& Stanford, S.S. 2003, ApJ, 585, 78

Woosley, S.E., \& Weaver, T.A. 1995, ApJS, 101, 181

Wuyts, S., et al. 2003, ApJ, 605, 677 